\title{
Psychological Intimate Partner Abuse among Chinese Women: What we know and what we still Need to know
}

\author{
Agnes Tiwari ${ }^{*}, 1$, Janet Wong ${ }^{1}$, Douglas A. Brownridge ${ }^{2}$, Ko Ling Chan ${ }^{3}$, Daniel Y. T. Fong ${ }^{1}$, \\ Wing Cheong Leung ${ }^{4}$, Pak Chnng $\mathrm{Ho}^{5}$ \\ ${ }^{I}$ Department of Nursing Studies, Li Ka Shing Faculty of Medicine, The University of Hong Kong, Hong Kong \\ ${ }^{2}$ Department of Family Social Sciences and Arthur V. Mauro Centre for Peace and Justice, University of Manitoba, \\ Winnipeg, Canada \\ ${ }^{3}$ Department of Social Work and Social Administration, The University of Hong Kong, Hong Kong \\ ${ }^{4}$ Department of Obstetrics and Gynaecology, Kwong Wah Hospital, Hong Kong \\ ${ }^{5}$ Department of Obstetrics and Gynaecology, Li Ka Shing Faculty of Medicine, The University of Hong Kong, Hong \\ Kong
}

\begin{abstract}
Although increasing attention has been given to psychological abuse in intimate partner relationships, relatively little is known about this complex problem among Chinese women. A better understanding of psychological abuse in Chinese intimate relationships is warranted because Chinese culture is shame-oriented. Women, therefore, may be more susceptible to shameful feelings induced by psychological abuse. This may explain why psychological abuse, in the absence of physical and/or sexual abuse, has such adverse mental health effects on Chinese women. In this paper, what is known about psychological abuse in Chinese intimate relationships and what still needs to be known are highlighted, together with the mental health impact. Implications for future research are also presented.
\end{abstract}

Keywords: Psychological abuse, intimate partner violence, Chinese women, prevalence, mental health, impact, shame, ridicule.

\section{INTRODUCTION}

Violence against women by an intimate partner is pervasive in society with negative consequences for women who experience it as well as for their children [1, 2]. Much of the research, however, has been on physical intimate partner abuse; psychological abuse has only recently been researched systematically [3]. Although there are still no adequate definitions of psychological abuse in intimate relationships, with advances in measurement there is now a better understanding of the nature and effects of psychological abuse in intimate relations $[4,5]$. Notwithstanding the encouraging development of research on psychological abuse, the recent efforts to address this social problem are mainly confined to Western literature. Relatively little is known about the nature, extent and impact of psychological intimate partner abuse among Chinese women. This is the current situation despite an earlier effort to devise and validate a Chinese version of the Index of Spouse Abuse (ISA) [6] as a measurement tool of psychological abuse in Chinese wives in Hong Kong [7]. The present paper aims to

*Address correspondence to this author at the Department of Nursing Studies, L9 Ka Shing Faculty of Medicine, The University of Hong Kong, 4/F, William MW Mong Block, 21, Sassoon Road, Pokfulam, Hong Kong; Tel: (852) 28192629 (O), (852) 92026616 (M); Fax: (852) 28726079 ; E-mail: afytiwar@hkucc.hku.hk raise awareness about psychological abuse in Chinese intimate relationships by highlighting what is known and what still needs to be known. The impact of psychological abuse on Chinese women is discussed and compared with that reported in Western literature. Implications for future research are also presented.

\section{PSYCHOLOGICAL ABUSE IN CHINESE INTIMATE RELATIONS}

Psychological abuse as a subtype of intimate partner violence (IPV) in Chinese women was first reported by Tang [8] in a population-based study conducted in 1996. Using the random digit dialing method to select respondents from the entire community in Hong Kong, Tang conducted telephone interviews with 1132 married women using the Conflict Tactic Scales (CTS) [9]. The prevalence of psychological abuse perpetrated by husbands during the surveyed year was $77.5 \%$ (compared with physical abuse which was $10 \%$ ). The most common form of psychological abuse was husbands sulking or refusing to talk $(56.7 \%)$. Subsequent populationbased studies conducted in Hong Kong and China found lower prevalence rates of psychological intimate partner abuse, ranging from 59\% in Chan's [10] household survey of 2706 women in Hong Kong using the Revised Conflict Tactics Scale (CTS2) [11] to 5\% in a survey of 687 women in Central China using self-constructed items [12]. 
In terms of research taking place within Chinese healthcare settings, the first study on psychological abuse was conducted in Hong Kong in 1998 [13]. Of the 631 pregnant women interviewed with the Abuse Assessment Screen (AAS) [14], 4.3\% reported being abused by their intimate partner during pregnancy and the abuse was mainly threats of violence without any physical injury (96.3\%). Subsequent studies conducted in healthcare settings in Hong Kong and China also showed variations in the prevalence rates of psychological abuse, as in the population-based studies. The highest prevalence rate was found in a survey of 1397 pregnant women in Central China, in which $9.1 \%$ reported psychological abuse during pregnancy based on selected CTS items [15]. The lowest rate was reported in a study of 500 women attending an outpatient fertility clinic in Hong Kong in which only $1 \%$ of the women reported psychological abuse when interviewed with the AAS [16].

The large variations in prevalence rates of psychological abuse across the population- and healthcare-based studies in China and Hong Kong are likely to be due to methodological differences. Specifically, none of these studies attempted to define psychological abuse. This problem is not unique to research on Chinese populations. There is little consensus on the definition of psychological abuse even in Western countries [17]. The lack of a clear conceptualization of psychological abuse as observed in most of the Chinese studies may account for much of the variation in prevalence rates since reported research may be measuring different aspects of psychological abuse.

In light of the lack of consensus on what constitutes psychological abuse in Chinese culture, the common practice of using measurement scales imported from Western nations with minimum efforts to assess their cross-cultural validity [18] should be questioned. Recently, an effort has been made to develop a valid and reliable tool for clinical screening of psychological abuse in Chinese women [19]. Modified from the AAS (14), the Chinese AAS is designed to address psychological and physical abuse separately for three time periods (lifetime, preceding 12 months and during pregnancy). This differs from the AAS which treats psychological and physical abuse simultaneously for the lifetime period while focusing only on physical and sexual abuse for the other time periods. To help respondents to decide if their partner's act constitutes psychological abuse, examples of psychologically abusive behaviors are provided. The examples are drawn from those elicited from Chinese women reporting psychological abuse in earlier studies [10, 13, 20, 21]. The examples include behaviors which cause denigrating damage to self-esteem, passive-aggressive withholding of emotional support and nurturance, threatening behaviors, and restricting personal territory and freedom, as described in Maiuro's [4] dimensions of psychological abuse. The Chinese AAS was validated with 257 abused and non-abused Chinese women and demonstrated satisfactory measurement accuracy and utility for identifying IPV including psychological abuse [19].

While the Chinese AAS may be a useful tool for clinical screening of psychological abuse, the question still remains as to what actually constitutes psychological maltreatment in Chinese relationships. Tolman, Rosen and Wood [5] suggest that it may be more useful to construe the behaviors on a continuum, with isolated hurtful behaviors on one end (and likely to be present to some degree in all relationships) and pervasive, one-sided, severe psychological torture on the other end. As well, a behavioral scale in itself may not be enough to make the delineation between distressed marital relationships and psychologically abusive relationships. Thus, there is a need to consider the context and motive of the act in order to decide what constitutes psychological abuse in the Chinese context (R.M. Tolman, personal communication, September 11, 2008). Interestingly, Chinese abused women themselves seem to have little problem in identifying whether their partner's behaviors are psychologically abusive or not, as we have found in our earlier studies $[10,13,20,21]$. Also, in a recent qualitative study to explore the contexts and consequences of psychological maltreatment of Chinese women, the accounts between abused women in a shelter and nonabused women in distressed marital relationships were clearly different in type, frequency, intensity, intent, reciprocity and effect ${ }^{1}$.

With the exception of a few studies [8, 10], the prevalence rates of psychological abuse in many of the Chinese studies are relatively low when compared to some Western studies [22, 23]. Therefore, it is worth exploring whether psychological abuse against Chinese women has the same impact as that observed in Western studies. In the following sections, the impact of psychological abuse on the mental health of Chinese women is discussed.

\section{MENTAL HEALTH IMPACT OF PSYCHOLOGICAL ABUSE ON CHINESE WOMEN}

In a study designed to explore the impact of wife abuse on the general and psychological well-being of Chinese women and their children in Hong Kong, Tang [24] found that husbands' verbal abuse, but not their physical abuse, was related to women's general distress including insomnia, anxious mood, and depression as measured by the General Health Questionnaire [25], the State Anxiety Inventory Form Y [26], and the Beck Depression Inventory [27]. As far as we can establish, this was the first study that reported explicitly on the impact of nonphysical abuse in Chinese intimate relationships.

The impact of verbal abuse on mental health was also found in Chinese pregnant women. In a prospective study involving a group of pregnant Chinese women in Hong Kong [20], verbal abuse was significantly related to postnatal depressive symptoms as measured by the Chinese version of the Edinburgh Postnatal Depression Scale (EPDS) [28]. More recently, in the first population-based survey of 3245 pregnant women in seven public hospitals in Hong Kong, women who reported psychological abuse, in the absence of physical and/or sexual abuse, had a higher risk of postnatal depression (as measured by the EPDS) and significantly poorer mental health-related quality of life (as measured by the SF-12 Health Survey) [29].

\footnotetext{
1 Tiwari A, Chan CH, Yuen KH, Salili F, Tang D. Differentiating psychological maltreatment between abused and non-abused Chinese women. Unpublished manuscript. Department of Nursing Studies, The University of Hong Kong 2008.
} 
The findings of the effects of psychological abuse in the Chinese studies are consistent with those reported in Western studies. In a general population survey conducted in the US, Straus, Sweet and Vissing [30] found that the more verbal aggression a woman experienced from her spouse, the more likely that she was depressed regardless of the presence of physical abuse. Similarly, Tolman and Bhosley [31] reported that psychological maltreatment was a powerful predictor of the victims' psychosocial problems. A more recent study also identified psychological intimate partner violence as a major predictor of posttraumatic stress disorder (PTSD) in abused women [32]. In a sample of sheltered and nonsheltered battered women, Sackett and Saunders [33] found that psychological abuse contributed to depression and low self-esteem, independent of physical abuse.

It has been suggested that various forms of psychological abuse may not have similar effects on battered women [34]. In a study of 234 abused women, Follingstad et al. [34] found that ridicule was the most negative form of psychological abuse, compared with jealousy, restriction, threats of abuse, threats of divorce and damage to property. It was postulated that ridicule attacked the women's sense of self-esteem and destroyed their ability to feel good about themselves. As a result, they felt helpless and worthless in their own eyes. The devastating effect of ridiculing was later confirmed by Sackett and Saunders [33] when it was shown to be related to depression. It was suggested that when the woman's partner ridiculed her about her trait, it was effectively an attack on her character and therefore more likely to shatter her sense of hope and security in her intimate relationship, thus leading to depression.

Similarly, ridicule was also identified as the worst form of intimate partner abuse by pregnant women in studies conducted in Hong Kong [10, 13, 21, 35]. As in the Western studies, ridiculing of abused victims' characters was also found to be related to depressive symptoms in Chinese women [21, 35]. Theoretically, the possible link between ridicule and depression may be explained in terms of Chinese cultural beliefs. Since traditional Chinese culture emphasizes the need for a woman to be a virtuous wife [18], ridiculing of traits by an intimate partner may make the woman feel diu lian (loss of face) for not living up to the expected standard [36]. Also, the rigid gender norms prescribed by Confucian decorum stipulate that women must remain submissive to their husbands [18]. When a Chinese woman is ridiculed by her husband about her personal character, she may experience can kui, which is the shameful feeling that one has failed to attain one's best or carry out a positive duty [36]. Furthermore, the rules of the three obediences in traditional Chinese culture require a woman to serve the needs of her husband [18]. However, ridicule undermines the woman's conceptualization of herself as a person and causes her to have xiu kui, which is a strong feeling of shame arising from the realization that she has harmed another person (in this case, her husband) because of her failing as a wife [36]. Finally, ridicule by an intimate partner can arouse $x i u$ chi, the strongest of the shameful emotions when the woman develops the feeling of having a hei dien (stain) on her face because of her inadequacy. Because of this hei dien, anyone who sees her will immediately know of her shame and condemn her [36]. Diu lian, can kui, xiu kui and xiu chi are types of shame in Chinese culture with varying degrees of debilitating effect on the person experiencing the feelings of shame [37]. In the Western studies, shame-proneness has been found to be strongly and positively related to psychopathological problems including depression, anxiety and somatization [38]. Street and Arias [39] also identified shame to be a mediating factor in the relationship between the emotional/ verbal form of psychological abuse and posttraumatic stress disorder. Thus, it is probable that shame emotions could be the link between ridicule and mental health problems (such as depression) in Chinese women. However, this is purely conjecture and empirical evidence is needed. Also, there are significant differences between Western and Eastern concepts of shame. For example, compared to their American counterparts, Chinese people are more sensitive to being personally shamed by actions (or lack of action) by other people [36]. Thus, caution should be exercised when making cross-cultural comparison of shame emotion.

The possible susceptibility of Chinese women to the various types of shame emotions may explain why psychological abuse would have such adverse effects on these women's mental health, even when there is no physical and/or sexual abuse, as observed in some of the Hong Kong studies [20, 21, 35]. Further efforts are needed to investigate the vulnerability of Chinese women to psychological abuse and enhance cross-cultural understanding. We now turn to implications for future research.

\section{IMPLICATIONS FOR FUTURE RESEARCH}

Methodological differences have so far prevented comparison of the findings of psychological intimate partner abuse across Chinese studies. In the absence of consistent and culturally relevant definitions of psychological abuse and its different forms across Chinese societies, crosscultural comparison is also not possible. Future research should address such methodological shortcomings by adopting strategies that would allow meaningful comparisons to be made among studies within China and across nations. The WHO Multicountry Study on Women's Health and Experiences [1] is a good example and many of the strategies (including the standardization of data collection and analyses to ensure comparability across study sites) are also relevant for psychological abuse research in Chinese populations.

Furthermore, there is a definite need to conduct longitudinal research on psychological intimate partner abuse in Chinese women. Since Western studies have shown that psychological abuse precedes physical abuse [3, 40], longitudinal research on psychological abuse in Chinese women over time is necessary to show if there is a progression from non-physical to physical abuse. It will also provide evidence on whether and how psychological abuse in Chinese intimate relationships differs from the development of physical or sexual abuse. Such information is important to inform the development and use of timely interventions for women experiencing the detrimental effects of psychological abuse in shame-oriented Chinese societies. 
Finally, further studies with representative populations are needed to establish a clear delineation between distressed marital relationships and psychologically abusive relationships in the Chinese context. This will help practitioners to conduct skillful assessment for psychological abuse and provide effective interventions in a culturally appropriate manner.

In conclusion, despite the emerging picture of psychological abuse in Chinese women, there is still a need to determine the difference between relationship distress and psychological abuse in intimate relationships. More attention should also be given to the mental health impact of psychological abuse on Chinese women to establish if they are indeed more vulnerable to this form of abuse.

\section{ACKNOWLEDGEMENTS}

We are indebted to Professor Richard Tolman for his expert guidance in helping us to understand the complexity of psychological abuse in Chinese women.

\section{REFERENCES}

[1] Garcia-Moreno C, Jansen HAFM, Ellsberg M, Heise L, Watts CH. Prevalence of intimate partner violence: findings from the WHO multi-country study on women's health and domestic violence. Lancet 2005; 368(9543): 1260-9.

[2] Krug EG, Mercy JA, Dahlberg LL, Zwi AB. World Report on Violence and Health. World Health Organization: Geneva, 2002.

[3] O'Leary KD. Psychological abuse: a variable deserving critical attention in domestic violence. Violence Vict 1999; 14(1): 3.

[4] Maiuro R. Sticks and stones may break my bones, but names will also hurt me: psychological abuse in domestically violence relationships. In: O'Leary $\mathrm{KD}$, Maiuro RD, Eds. Psychological abuse in violent domestic relations (pp ix-xx). Springer Publishing Company: New York, 2001.

[5] Tolman R, Rosen D, Wood GC. Assessment of psychological maltreatment. In: Ammerman R, Hersen M, Eds. Assessment of family violence: A clinical and legal sourcebook. 2nd ed. John Wiley and Sons: New York, 1999.

[6] Hudson W, McIntosh S. The assessment of spouse abuse: two quantifiable dimensions. J Marriage Fam 1981; 43(4): 873-85.

[7] Tang C. Psychological abuse of chinese wives. J Fam Violence 1998; 13(3): 299-314

[8] Tang CS-k. Wife abuse in hong kong chinese families: a community survey. J Fam Violence 1999; 14(2): 173-91.

[9] Straus M. Measuring intrafamily conflict and violence: the conflict tactics scales (CTS). J Marriage Fam 1979; 41(1): 75-88.

[10] Chan K. Study on Child Abuse and Spouse Battering: Report on Findings of Household Survey [A Consultancy Study Commissioned By the SWD of the HKSAR Government] Department of Social Work \& Social Administration. The University of Hong Kong: Hong Kong, 2005.

[11] Straus M, Hamby S, Boney-McCoy S, Sugarman D. The revised conflict tactics scales (CTS2): development and preliminary psychometric data. J Fam Issues 1996; 17(3): 283.

[12] Li X, Ching Y, Hwang N. A survey of domestic violence in rural China. Chin Behav Med Sci 2003; 12: 228-30 (in Chinese).

[13] Leung WC, Leung TW, Lam YY, Ho PC. The prevalence of domestic violence against pregnant women in a Chinese community. Int J Gynaecol Obstet 1999; 66(1): 23-30.

[14] McFarlane J, Parker B, Soeken K, Bullock L. Assessing for abuse during pregnancy. Severity and frequency of injuries and associated entry into prenatal care. JAMA 1992; 267(23): 3176-8.

[15] Ye ZH, Wang SY, Xiao XM, et al. A study of domestic violence in pregnancy and post-partum. Chin J Public Health 2005; 21: 1012-3.

[16] Leung T, Ng E, Leung W, Ho P. Intimate partner violence among infertile women. Int J Gynecol Obstet 2003; 83(3): 323-4.
[17] Schumacher J, Feldbau-Kohn S, Smith Slep A, Heyman R. Risk factors for male-to-female partner physical abuse. Aggres Violent Behav 2001; 6(2-3): 281-352.

[18] Tang C, Lai B. A review of empirical literature on the prevalence and risk markers of male-on-female intimate partner violence in contemporary China, 1987-2006. Aggress Violent Behav 2008; 13(1): 10-28

[19] Tiwari A, Fong DY, Chan KL, Leung WC, Parker B, Ho PC. Identifying intimate partner violence: comparing the chinese abuse assessment screen with the chinese revised conflict tactics scales. BJOG 2007; 114(9): 1065-71.

[20] Leung WC, Kung F, Lam J, Leung TW, Ho PC. Domestic violence and postnatal depression in a Chinese community. Int J Gynecol Obstet 2002; 79(2): 159-66.

[21] Tiwari A, Leung WC, Leung TW, Humphreys J, Parker B, Ho PC. A randomised controlled trial of empowerment training for Chinese abused pregnant women in Hong Kong. BJOG 2005; 112(9): 124956.

[22] Follingstad D, DeHart DD. Defining psychological abuse of husbands toward wives: contexts, behaviors, and typologies. J Interpers Violence 2000; 15(9): 891-920.

[23] Stets J. Verbal and physical aggression in marriage. J Marriage Fam 1990; 52(2): 501-14.

[24] Tang C. Psychological impact of wife abuse: experiences of chinese women and their children. J Interp Violence 1997; 12(3): 466.

[25] Goldberg DP. The detection of psychiatric illness by questionnaire. Mansley Monograph No. 21, University Press; Oxford: London, 1972

[26] Spielberger CD, Gorsuch RC, Lushene RE. Manual for the state trait anxiety inventory. Consulting Psychologists Press: Palo Alto, CA 1970.

[27] Beck A, Ward C, Mendelson M, Mock J, Erbaugh J. An inventory for measuring depression. Arch Gen Psychiat 1961; 4: 561-71.

[28] Lee DT, Yip SK, Chiu HF, et al. Detecting postnatal depression in Chinese women. Validation of the Chinese version of the edinburgh postnatal depression scale. Br J Psychiat 1998; 172(5): 433-7.

[29] Ware JE, Kosinski M, Keller SD. A 12 item short form health survey: construction of scales and preliminary tests of reliability and validity. Med Care 1996; 34: 220-33.

[30] Straus M, Sweet S, Vissing Y. Verbal aggression against spouses and children in a nationally representative sample of American families. Paper presented at the Annual Meeting of the Speech Communication Association, San Francisco, CA 1989.

[31] Tolman R, Bhosley G. The outcome of participation in sheltersponsored program for men who batter. In: Knudsen D, Miller J, Eds. Abused and battered: Social and legal responses to family violence (PP 113-122). New York: Aldine de Gruyter; 1991.

[32] Pico-Alfonso MA. Psychological intimate partner violence: the major predictor of posttraumatic stress disorder in abused women. Neurosci Biobehav Rev 2005; 29(1): 181-93.

[33] Sackett LA, Saunders DG. The Impact of different forms of psychological abuse on battered women. Violence Vict 1999; 14: 105-17.

[34] Follingstad DR, Rutledge LL, Berg BJ, Hause ES, Polek DS. The role of emotional abuse in physically abusive relationships. J Fam Violence 1990; 5(2): 107-20.

[35] Tiwari A, Chan KL, Fong D, et al. The impact of psychological abuse by an intimate partner on the mental health of pregnant women. BJOG 2008; 115(3): 377-84.

[36] Bedford OA. The individual experience of guilt and shame in chinese culture. Culture Psychol 2004; 10(1): 29-52.

[37] Bedford O, Hwang KK. Guilt and shame in chinese culture: a cross-cultural framework from the perspective of morality and identity. J Theory of Soc Behav 2003; 33(2): 127-44.

[38] Tangney J, Wagner P, Gramzow R. Proneness to shame, proneness to guilt, and psychopathology. J Abnormal Psychol 1992; 101(3): 469-78.

[39] Street AE, Arias I. Psychological abuse and posttraumatic stress disorder in battered women: examining the roles of shame and guilt. Violence Vict 2001; 16(1): 65-78. 
[40] Schumacher J, Leonard K. Husbands' and wives' marital adjustment, verbal aggression, and physical aggression as longitudinal predictors of physical aggression in early marriage. $\mathrm{J}$ Consult Clin Psychol 2005; 73(1): 28-37.

\section{(C) Agnes Tiwari; Licensee Bentham Open}

This is an open access article licensed under the terms of the Creative Commons Attribution Non-Commercial License (http: //creativecommons.org/licenses/bync/3.0/), which permits unrestricted, non-commercial use, distribution and reproduction in any medium, provided the work is properly cited. 